\title{
Interplay of variable thermal conductivity and expansivity on the thermal structure of oceanic lithosphere II
}

\author{
S. Honda ${ }^{1}$ and D. A. Yuen ${ }^{2}$ \\ ${ }^{1}$ Earthquake Research Institute, University of Tokyo, 1-1-1 Yayoi, Bunkyou-ku, Tokyo 113-0032, Japan \\ ${ }^{2}$ Minnesota Supercomputer Institute, University of Minnesota, Minneapolis, MN 55455, U.S.A.
}

(Received January 17, 2004; Revised March 16, 2004; Accepted March 25, 2004)

\begin{abstract}
We have extended our previous analysis of the effects of constant vs. variable, i.e., pressure and temperature dependent thermal conductivity $(k)$ and constant thermal expansivity $(\alpha)$ on the thermal structure of the oceanic lithosphere. We apply our analysis to the actual data set including information on the geoid slope. The heat flow and ocean floor depth data constrain the thermal expansivity $\left(\alpha \approx 3 \times 10^{-5} 1 /{ }^{\circ} \mathrm{C}\right)$. Including geoid slope data may loosely constrain both the thermal expansivity and the thermal conductivity. The probable value of thermal conductivity is $\approx 3 \mathrm{~W} / \mathrm{m} /{ }^{\circ} \mathrm{C}$ for the constant $k$ case and $\approx 4 \mathrm{~W} / \mathrm{m} /{ }^{\circ} \mathrm{C}$ (at ambient conditions) for the variable $k$ case. These $\alpha$ and $k$ are generally consistent with laboratory data of appropriate lithospheric materials. Our analysis supports the plate model with thin lithosphere and high bottom temperature, such as GDH1 $\left(95 \mathrm{~km} ; 1450^{\circ} \mathrm{C}\right)$. Variable $k$ case requires slightly thinner and higher temperature lithosphere $\left(\approx 85 \mathrm{~km}\right.$ and $\left.\approx 1500^{\circ} \mathrm{C}\right)$ and gives a slightly better fit to the geoid slope data.
\end{abstract}

Key words: Thermal structure of oceanic lithosphere, thermal conductivity, thermal expansivity.

\section{Introduction}

Studies of the thermal structure of oceanic lithosphere originates in 1960's (e.g., McKenzie, 1967; Turcotte and Oxburgh, 1967). From these studies, two currently popular thermal models of oceanic lithosphere, i.e. cooling halfspace model (Turcotte and Oxburgh, 1967) and plate model (McKenzie, 1967) emerged. These models have been tested or constrained mainly by heat flow and ocean floor depth data (e.g., Sclater et al., 1981). Recently, based on an extensive compilation of heat flow and ocean floor depth data, Stein and Stein (1992) proposed the thinner and hotter plate (GDH1 model) than that of the previous model by Parsons and Sclater (1977). Geoid slope data are also found to be useful to constrain the thermal model of plates (e.g., see DeLaughter et al. (1999) and references there in).

Thermal structure of the oceanic lithosphere is strongly controlled by the initial and boundary conditions which are reflected in the thickness and the bottom temperature of plate. However, it is also controlled by the physical properties of plate constituting material, i.e. density, heat capacity, thermal conductivity and thermal expansivity. Doin and Fleitout (1996) allowed a variation of thermal conductivity and expansivity around the experimentally determined values in their inversion of the CHABLIS model (See Section 4). Ideally, those physical properties should be constrained by the data only. However, probably this is not the case, especially when we use the heat flux and ocean floor depth data only. Honda and Yuen (2001) partially solved this problem using the work of pressure and temperature

Copy right (C) The Society of Geomagnetism and Earth, Planetary and Space Sciences (SGEPSS); The Seismological Society of Japan; The Volcanological Society of Japan; The Geodetic Society of Japan; The Japanese Society for Planetary Sciences; TERRAPUB. dependence of thermal conductivity by Hofmeister (1999). However, since they were rather interested in the differences of the thermal structure of the lithosphere caused by the pressure- and temperature-dependence of thermal conductivity, they used the theoretical output from GDH1 from Stein and Stein (1992) as model constraints. Also, they did not take into account the geoid slope data, which may support the thin and hot lithosphere (e.g., DeLaughter et al., 1999; Richardson et al., 1995).

Heat flux, ocean floor depth and geoid data have different sensitivities on the thermal structure of the lithosphere. Heat flux is just a temperature gradient at the top surface, the ocean floor depth is proportional to the depth-integrated temperature and the geoid is the weighted depth-integral of temperature (e.g. Richardson et al., 1995; Cazenave, 1984). Combining these different data sets may constrain the physical properties of lithospheric materials and eventually the thermal structure of the oceanic lithosphere.

In this paper, we extend Honda and Yuen's (2001) analysis using the real data sets and including the geoid slope data in order to see how they constrain the physical parameters (i.e., thermal conductivity and expansivity).

\section{Analysis}

The method of analyses is just the same as that of Honda and Yuen (2001) and it is forward modeling. The model is one-dimensional heat conduction, whose governing Eq. is given by

$$
\rho C_{p} \frac{\partial T}{\partial t}=\frac{\partial}{\partial z}\left(k \frac{\partial T}{\partial z}\right)
$$

where $T$ is the temperature $\left({ }^{\circ} \mathrm{C}\right), \rho=3300\left(\mathrm{~kg} / \mathrm{m}^{3}\right.$ : fixed value) is the mantle density, $C_{p}=1.17\left(\mathrm{KJ} / \mathrm{kg} /{ }^{\circ} \mathrm{C}\right.$ : fixed value) is the specific heat, $t$ is the age of ocean floor, and 
$z$ is the depth measured from the bottom of the ocean floor (positive upward). $k$ is either constant $\left(=k_{0}\right)$ or given by

$$
\begin{aligned}
k & =k_{0}\left(\frac{298}{T}\right)^{a} \exp \left[-\left(4 \gamma+\frac{1}{3}\right) \alpha(T-298)\right]\left(1+\frac{K_{T}^{\prime} P}{K_{T}}\right) \\
& +0.01 .753-0.00010365 T+\frac{2.2451 T^{2}}{10^{7}}-\frac{3.407 T^{3}}{10^{11}}
\end{aligned}
$$

where $P$ is the pressure, $k_{0}$ is the lattice thermal conductivity at ambient conditions $\left(25^{\circ} \mathrm{C}, 1 \mathrm{~atm}\right), \alpha$ is the thermal conductivity, $\gamma(=1.2)$ is the Grünneisen parameter, $K_{T}(=135$ $\mathrm{GPa})$ is the bulk modulus, $K_{T}^{\prime}(=4)$ is the pressure derivative of the bulk modulus and $a(=0.33$ : a typical value of silicates) is a constant (Hofmeister, 1999). In Eq. (2), $T$ is the absolute temperature. $\alpha$ is taken to be constant for simplicity, though it is treated as a parameter. This formulation includes both lattice conduction (the first term) and the radiative heat transfer (remaining terms). The boundary conditions are $T(z, 0)=T(-D, t)=T_{m}$ and $T(0, t)=0$, where $D$ and $T_{m}$ are the thickness of the plate and the temperature at the ridge and at the bottom of the plate. This model can naturally include the cooling half space model, if $D$ is set to be very large value. It can be shown that the plate model with a constant speed (McKenzie, 1967) is equivalent to this one-dimensional heat conduction model by assuming that the horizontal temperature gradient is small and the distance from the ridge is converted to the age. We also point out that this kind of models depends on the steadiness of plate movement.

A finite difference version of Eq. (1) (forward in time and central difference for diffusion term) is numerically integrated for given sets of $T_{m}, D, \alpha$ and $k_{0}$. The spatial resolution of the lithospheric domain is $1 \mathrm{~km}$ and small enough time-stepping is employed for numerical stabilization of the non-linear diffusion equation.

The model can be compared with the observations, that is, heat flux $Q(t)$, ocean floor depth $d(t)$ and geoid slope $g s(t)$, which is defined by $d(g e o(t)) / d t$ where $g e o(t)$ is the geoid height at age $t$. They can be predicted from the models by $Q(t)=-k \partial T / \partial z$ at $\mathrm{z}=0, d(t)=\frac{\rho \alpha}{\left(\rho-\rho_{w}\right)} \int_{-D}^{0}(T(z, t)-$ $\left.T_{m}\right) d z\left(\rho_{w}=1000 \mathrm{~kg} / \mathrm{m}^{3}\right.$ : the density of sea water $)$ and $g s(t)=\frac{2 \pi G}{g} \frac{\partial}{\partial t} \int_{-D}^{0} z \Delta \rho(z) d z\left(G=6.6732 \times 10^{-11} \mathrm{Nm}^{2}\right.$ $\mathrm{kg}^{-2}$ : gravitational constant, $g=9.8 \mathrm{~m} / \mathrm{s}^{2}$ reference acceleration of gravity $\Delta \rho$ : density difference at $z$ between the mantle under the ridge and the ocean floor of the age $t$. See, for example, Turcotte and Schubert, 1982).

We have three types of misfits between model predictions and observations defined by

$$
\delta_{T Y P E}^{2}=\frac{1}{N_{T Y P E}} \sum \frac{\left(T Y P E_{O B S}-T Y P E_{M O D E L}\right)^{2}}{\delta_{T Y P E, O B S}^{2}}
$$

where "TYPE" is either $Q$ (heat flux), $d$ (ocean floor depth) or $g s$ (geoid slope). The suffix "OBS" and "MODEL" imply "observations" and "model output", respectively. $\delta_{T Y P E, O B S}^{2}$ is the square of standard error of the data of "TY PE". The summation is made for a total number of data $\left(N_{T Y P E}\right)$. To evaluate the model fits, we use two types of misfits, that is,

$$
\delta_{Q d}^{2}=\left(\delta_{Q}^{2}+\delta_{d}^{2}\right) / 2
$$

and

$$
\delta_{Q d g s}^{2}=\left(\delta_{Q}^{2}+\delta_{d}^{2}+\delta_{g s}^{2}\right) / 3 .
$$

Data sets are from the compilation of Kido and Seno (1994) for ocean floor depth, Stein and Stein (1992) for heat flux and Richardson et al. (1995) for geoid slope.

The parameters of our models are $k_{0}, \alpha, T_{m}$ and $D$, and they are changed, systematically, from 2.5 to $6.0\left(\mathrm{~W} / \mathrm{m} /{ }^{\circ} \mathrm{C}\right)$, $2 \times 10^{-5}$ to $4.5 \times 10^{-5}\left(1 /{ }^{\circ} \mathrm{C}\right), 1000$ to $1800\left({ }^{\circ} \mathrm{C}\right)$ and 60 to 125 $(\mathrm{km})$ at increments of $0.5\left(\mathrm{~W} / \mathrm{m} /{ }^{\circ} \mathrm{C}\right), 0.5 \times 10^{-5}\left(1 /{ }^{\circ} \mathrm{C}\right), 50$ $\left({ }^{\circ} \mathrm{C}\right)$ and $50(\mathrm{~km})$, respectively. For $\alpha$ we make an additional calculation with $\alpha=2.25 \times 10^{-5}\left(1 /{ }^{\circ} \mathrm{C}\right)$.

\section{Results}

By fixing the physical parameters, $\alpha$ and $k_{0}$, we can determine the combination of $D$ and $T_{m}$ which gives the smallest $\delta_{Q d g s}^{2}$ or $\delta_{Q d}^{2}$. The obtained results by this way are summarized in Fig. 1 for constant $k$ and Fig. 2 for variable $k$. The left and right columns of Figs. 1 and 2 show $\delta_{Q d}^{2}$ and $\delta_{Q d g s}^{2}$, respectively. They are normalized by the smallest values of each $\delta^{2}$ (We denote this $\overline{\delta^{2}}$, that is, $\overline{\delta_{T Y P E}^{2}}=$ $\left.\delta_{T Y P E}^{2} / \min \left(\delta_{T Y P E}^{2}\right)\right)$, and subtracted 1 . The radius of filled circles is proportional to their magnitudes. The solid and dashed lines show iso- $\alpha$ and $k_{0}$ lines, respectively and their values are show by normal characters $\left(k_{0}\right.$ : see the topmost figures of Figs. 1 and 2) and bold italic characters ( $\alpha$ : see the middle figures of Figs. 1 and 2), respctively.

We also show the estimates of Parsons and Sclater's (1977) model ("P") and GDH1 ("G") (see the lowermost left figures). Thick dashed lines in all figures are the solidi from McKenzie and Bickle (1988).

In the topmost figures, if $\overline{\delta^{2}}$ exceeds 2 (shown by "Tcut" in Figs. 1 and 2), we set $\overline{\delta^{2}}$ to 2 . From these results, one may notice that the combination of the heat flow and ocean floor depth data cannot constrain the range of $k$ but it may constrain the range of $\alpha$. On the other hand, an inclusion of geoid data appears to constrain the range of both $\alpha$ and $k$. However, the resultant residuals $\delta_{Q d g s}^{2}$ are usually a several times larger than $\delta_{Q d}^{2}$, and the lower limit of $k$ appears not to be well costrained.

If we know the right weighting for $\delta^{2}$ of different type (i.e., heat flux, ocean floor depth and geoid slope) of data sets, we may determine a reasonable estimate of $\alpha$ and $k$ using a single residual map. However, since this is not the case, we evaluate the appropriateness of parameters by making additional checks of the fit of each data type.

In the middle and bottom sections of Figs. 1 and 2, we set $\overline{\delta_{Q d}^{2}}$ and $\overline{\delta_{Q d g s}^{2}}$ to 2 , if either $\overline{\delta_{Q}^{2}}, \overline{\delta_{d}^{2}}$ or $\overline{\delta_{g s}^{2}}$ exceeds the threshold values which are shown in Figs. 1 and 2 by "Ecut". As the threshold values decrease, $\overline{\delta^{2}}$ of some combinations of parameters becomes 2 , and, thus, they can be excluded from appropriate parameter combinations.

A combination of heat flow and ocean floor depth data gives a rather broad minimum and the change of residual distribution associated with the change of threshold value is not systematic. This may occur because this combination cannot restrict $k$. On the other hand, an inclusion of geoid slope data narrows the range of appropriate parameters, systematically as the threshold decreases. It may be notable that the parameter combination close to GDH1 model for constant $k$ 

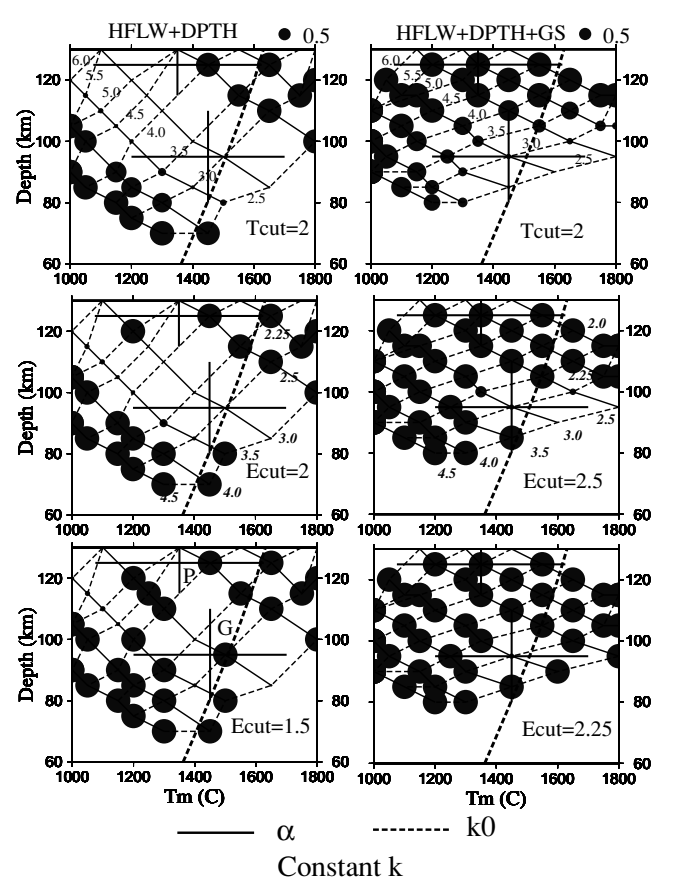

Fig. 1. The combination of $D$ and $T_{m}$ which gives the least residuals for given $\alpha$ and $k_{0}$ for constant thermal conductivity. The radius of the filled circles show the magnitude of $\overline{\delta^{2}}-1$. The radius of the filled circles shown on the right corner is 0.5 . Left and right columns show the $\left.\overline{\left(\delta_{Q d}^{2}\right.}-1\right)$ and $\left.\overline{\left(\delta_{Q d g s}^{2}\right.}-1\right)$, respectively. Solid and dashed lines connect the points with equi- $\alpha$ and $k_{0}$, respectively. Numerals in the top figures show the values of $k_{0}\left(\mathrm{~W} / \mathrm{m} /{ }^{\circ} \mathrm{C}\right)$. Numerals shown by bold italics in the middle figures show the values of $\alpha \times 10^{5}\left(1 /{ }^{\circ} \mathrm{C}\right)$. See text for detail.

case survives until the end (Fig. 1, bottom right). However, generally the range of appropriate parameter combinations is elongated toward the constant $\alpha$ direction.

As discussed before (Honda and Yuen, 2001), the heat flux $Q$ and ocean floor depth $d$ may be scaled by $Q \propto k T_{m} / D$ and $d \propto \alpha D T_{m}$. The trend of the minimum $\overline{\delta_{Q d}^{2}}$ looks a hyperbola in top left diagrams of Figs. 1 and 2. This implies that the fit is controlled by the ocean floor depth suggesting that the models are more sensitive to the ocean floor depth than to the heat flux.

From above results, we estimate $\alpha \approx 3 \times 10^{-5}(1 / \mathrm{C})$ and $k_{0} \approx 3(\mathrm{~W} / \mathrm{m} / \mathrm{C})$ for constant $k$ and $k_{0} \approx(3-4)(\mathrm{W} / \mathrm{m} / \mathrm{C})$ for variable $k$. These estimates are generally consistent with laboratory measurements of appropriate mantle materials (e.g., see Fei, 1995; Hofmeister, 1999).
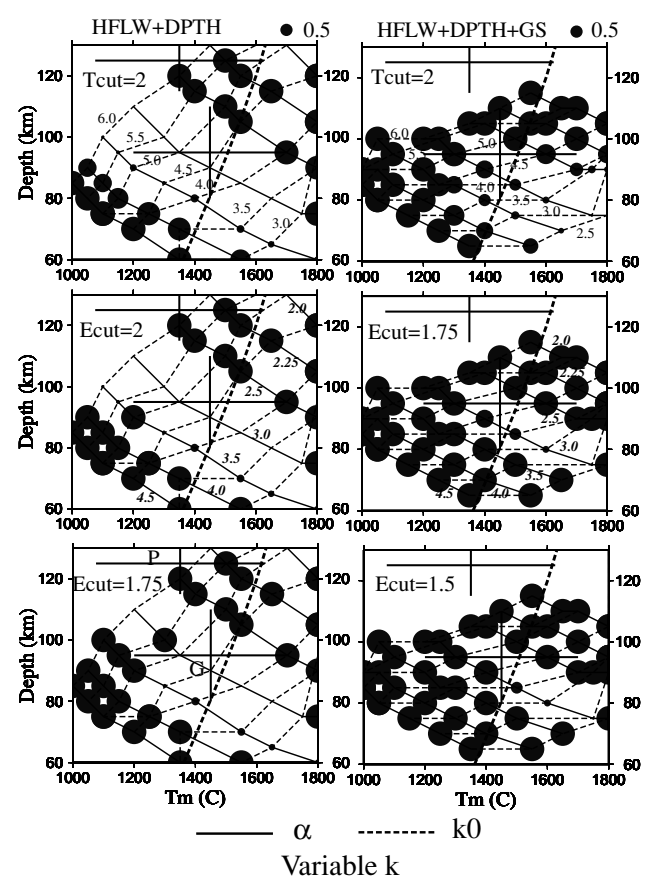

Fig. 2. The combination of $D$ and $T_{m}$ which gives the least residuals for given $\alpha$ and $k_{0}$ for variable thermal conductivity. See the caption of Fig. 1.

\section{Discussions}

Based on the present analysis, we choose a model which gives a comparable fit to data as GDH1 does and compare them. This model, which we call " $\mathrm{V}$ ", is characterized by the parameters $\alpha=3 \times 10^{-5}\left(1 /{ }^{\circ} \mathrm{C}\right), k_{0}=4\left(\mathrm{~W} / \mathrm{m} /{ }^{\circ} \mathrm{C}\right)$ (variable thermal conductivity), $D=85(\mathrm{~km})$ and $T_{m}=1500\left({ }^{\circ} \mathrm{C}\right)$. Misfits of each model are given in Table 1 . We choose the case with $k_{0}=4$ rather than 3 , since $k_{0}=3$ gives higher temperature than $k_{0}=4$ implying extensive melting (See Fig. 2).

Figure 3 shows graphical presentations of data fits. Both GDH1 (solid lines) and V (dashed lines) show similar fits, although the geoid slope of $\mathrm{V}$ gives a slightly better fit to the data near the ridge. This occurs since the model $\mathrm{V}$ cools slower than GDH1 does. This is evident in Fig. 4 of temperature distribution versus the age of plate. The slow cooling may occur partly because of the temperature dependence of $k$, that is, $k$ decreases as the temperature increases.

Doin and Fleitout (1996) proposed the CHABLIS model, in which the heat flux at given temperature is fixed. The
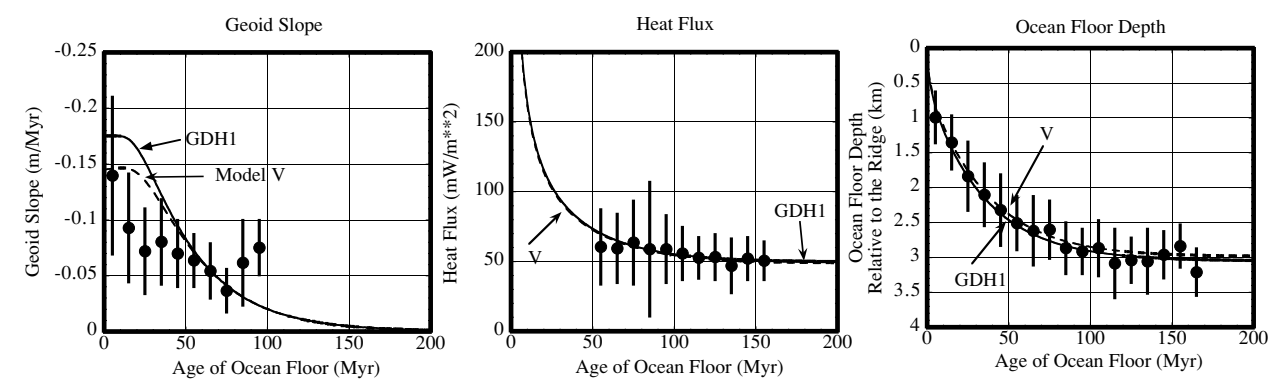

Fig. 3. Data fit of model GDH1 (Solid lines) and V (Dashed lines). From the left, they are (1) geoid slope (Richardson et al., 1995), (2) heat flux (Stein and Stein, 1992) and (3) ocean floor depth (Kido and Seno, 1994). 
Table 1. Summary of models. Also shown are the minimum residuals for constant $k$ (column of GDH1) and variable $k$ (column of $\mathrm{V}$ ). They are shown in the brackets.

\begin{tabular}{ccc}
\hline & GDH1 & $\mathrm{V}$ \\
\hline$D(\mathrm{~km})$ & 95 & 85 \\
$T_{m}\left({ }^{\circ} \mathrm{C}\right)$ & 1450 & 1500 \\
$\alpha\left(1 /{ }^{\circ} \mathrm{C}\right)$ & $3.1 \times 10^{-5}$ & $3 \times 10^{-5}$ \\
$k_{0}\left(\mathrm{~W} / \mathrm{m} /{ }^{\circ} \mathrm{C}\right)$ & 3.25 & 4 \\
$\delta_{d}^{2}$ & $0.0719(0.0709)$ & $0.0914(0.0715)$ \\
$\delta_{Q}^{2}$ & $0.0246(0.0177)$ & $0.0234(0.0177)$ \\
$\delta_{g s}^{2}$ & $1.42(0.355)$ & $1.02(0.360)$ \\
$\delta_{Q d}^{2}$ & $0.0483(0.0492)$ & $0.0574(0.0505)$ \\
$\delta_{Q d g s}^{2}$ & $0.505(0.352)$ & $0.379(0.255)$ \\
\hline
\end{tabular}

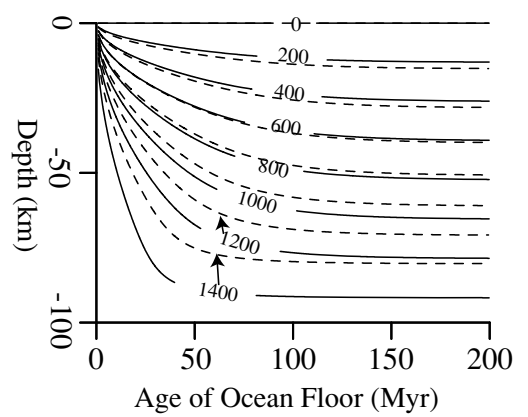

Fig. 4. Comparison between the constant (solid lines: GDH1) and variable (dashed lines) thermal conductivity for thermal structure of plate. GDH1 is characterized by $D=95(\mathrm{~km}), T_{m}=1450\left({ }^{\circ} \mathrm{C}\right), \alpha=3.1 \times 10^{-5}$ $\left(1 /{ }^{\circ} \mathrm{C}\right)$ and $k=3.1\left(\mathrm{~W} / \mathrm{m} /{ }^{\circ} \mathrm{C}\right)$. Model $\mathrm{V}$ is characterized by $D=85$ $(\mathrm{km}), T_{m}=1500\left({ }^{\circ} \mathrm{C}\right), \alpha=3.0 \times 10^{-5}\left(1 /{ }^{\circ} \mathrm{C}\right)$ and $k_{0}=4\left(\mathrm{~W} / \mathrm{m} /{ }^{\circ} \mathrm{C}\right)$.

underlying philosophy of their model is a ubiquitous occurrence of small-scale convection beneath the oceanic lithosphere. They found that their model also gives as good fit to the data as GDH1 does, although their thermal expansivity is higher $\left(\approx 4 \times 10^{-5}\right)$ than that of GDH1 and our analysis $\left(\approx 3 \times 10^{-5}\right)$. Their model also gives a slightly better fit to the geoid slope data, since it cools slower than the GDH1 does.

Another important point of thermal field is that the temperature difference between GDH1 and V is quite large (It may reach $\approx 100$ degrees at $\approx 80 \mathrm{~km}$ deep), although they give a similar fit to data. Such a large difference in the temperature will cause a large viscosity difference and it may affect the onset of small-scale convection beneath the lithosphere (Yanagawa, 2004).

Our analysis supports a thin $(\approx 90 \mathrm{~km})$ and hot $\left(\approx 1500^{\circ} \mathrm{C}\right)$ plate model for the cooling oceanic lithosphere. This becomes most evident, if we include the geoid slope data, even under the condition that we do not fix $k$ and $\alpha$ apriori. Also, the geoid slope data may have a possibility to discriminate either a constant $k$ or variable $k$. However, unfortunately, the uncertainty of geoid slope data is large and its model fit is poor compared to other type of data. In order to get a concrete answer, we need more careful analysis of geoid slope data.

We also note that, since the preferred plate model shows a fairly high temperature in the mantle, we may expect a partial melting, at least, at younger age. This may occur especially for variable $k$ case. However, the CHABLIS model of Doin and Fleitout (1996) predicts rather low temperature at the base of plate, which is equivalent to $T_{m}$, since it assumes the additional heat flux from below. This implies that the thermal structure may depend also on the model assumptions. Thus, although we believe that a simple model like the one presented here will help understanding a general characteristic of the thermal structure of the oceanic lithosphere, in order to estimate the precise thermal structure, we need to study models, which incorporate the partial melting (e.g. Kono and Yoshii, 1975), and eventually fully dynamical model (Dumoulin et al., 2001).

Acknowledgments. We thank Makoto Yamano and Anne Hofmeister for their constructive comments. This work has come out from the Japan (JSPS)-US (National Science Foundation) cooperative program.

\section{References}

Cazenave, A., Thermal cooling of the lithosphere: constraints from geoid data, Earth Planet. Sci. Lett., 70, 395-406, 1984.

DeLaughter, J., S. Stein, and C. A. Stein, Extraction of a lithospheric cooling signal from oceanwide geoid data, Earth Planet. Sci. Lett., 174, 173-181, 1999.

Doin, M. P. and L. Fleitout, Thermal evolution of the oceanic lithosphere, Earth Planet. Sci. Lett., 142, 121-136, 1996.

Dumoulin, C., M.-P. Doin, and L. Fleitout, Numerical simulations of an oceanic lithosphere above a convective mantle, Phys. Earth Planet. Int., 125, 45-64, 2001.

Fei, Y., Thermal expansion, in AGU Reference Shelf 2, Mineral Physics and Crystallography, A Handbook of Physical Constants, edited by T. J. Ahrens, pp. 29-44, American Geophysical Union, Washington D. C., 1995.

Hofmeister, A. M., Mantle values of thermal conductivity and the geotherm from phonon lifetimes, Science, 283, 1699-1706, 1999.

Honda, S. and D. A. Yuen, Interplay of variable thermal conductivity and expansivity on the thermal structure of oceanic lithosphere, Geophys. Res. Lett., 28, 351-354, 2001.

Kido, M. and T. Seno, Dynamic topography compared with residual depth anomalies in oceans and implications for age-depth curves, Geophys. Res. Lett., 21, 717-720, 1994.

Kono, Y. and T. Yoshii, Numerical experiments on the thickening plate model, J. Phys. Earth., 23, 63-75, 1975.

McKenzie, D., Some remarks on heat flow and gravity anomalies, J. Geophys. Res., 72, 6261-6273, 1967.

McKenzie, D. and M. J. Bickle, The volume and compaction of melt generated by extension of the lithosphere, J. Petrol., 29, 625-679, 1988.

Parsons, B. and J. G. Sclater, An analysis of the variation of ocean floor bathymetry and heat flow with age, J. Geophys. Res., 82, 803-827, 1977.

Richardson, W. P., S. Stein, C. A. Stein, and M. T. Zuber, Geoid data and thermal structure of the oceanic lithosphere, Geophys. Res. Lett., 22, 1913-1916, 1995.

Sclater, J. G., C. Jaupart, and D. Galson, The heat flow through oceanic and continental crust and the heat loss of the earth, Rev. Geophys. Space Phys., 18, 269-311, 1981.

Stein, C. A. and S. Stein, A model for the global variation in oceanic depth and heat flow with lithospheric age, Nature, 359, 123-129, 1992.

Turcotte, D. L. and E. R. Oxburgh, Finite amplitude convective cells and continental drift, J. Fluid Mech., 28, 29-42, 1967.

Turcotte, D. L. and G. Schubert, Geodynamics: Applications of Continuum Physics to Geological Problems, pp. 450, John Wiley and Sons, New York, 1982.

Yanagawa, T., Influence of temperature-dependnet thermal conductivity on mantle convection, Ph.D. Thesis, Kyushu University, 2004.

S. Honda (e-mail: honda@eri.u-tokyo.ac.jp) and D. A. Yuen (e-mail: davey@krissy.geo.umn.edu) 